\title{
Seminario internacional: «Jerarquías urbanas: ¿una pirámide trunca?»
}

\section{Alice Beuf}

\section{(2) OpenEdition}

\section{Journals}

Edición electrónica

URL: http://journals.openedition.org/bifea/4106

DOI: $10.4000 /$ bifea.4106

ISSN: 2076-5827

Editor

Institut Français d'Études Andines

Edición impresa

Fecha de publicación: 1 agosto 2013

Paginación: 313-317

ISSN: 0303-7495

\section{Referencia electrónica}

Alice Beuf, « Seminario internacional: «Jerarquías urbanas: ¿una pirámide trunca?» », Bulletin de I'Institut français d'études andines [En línea], 42 (2) | 2013, Publicado el 08 agosto 2013, consultado el 06 noviembre 2020. URL : http://journals.openedition.org/bifea/4106 ; DOI : https://doi.org/10.4000/ bifea.4106

\section{(c) $($ ) $\odot$}

Les contenus du Bulletin de l'Institut français d'études andines sont mis à disposition selon les termes de la licence Creative Commons Attribution - Pas d'Utilisation Commerciale - Pas de Modification 4.0 International. 
SEMINARIO INTERNACIONAL: «JERARQUÍAS URBANAS: ¿UNA PIRÁMIDE TRUNCA?»

\section{Quito, 3 y 4 de junio de 2013}

El Programa de Políticas Urbanas y Desarrollo de Ciudades (PRO.POLIS) de la Escuela de Gobierno y Administración Pública (EGAP) del Instituto de Altos Estudios Nacionales de Ecuador (IAEN), junto al Instituto Francés de Estudios Andinos (IFEA), la Cooperación Regional para los Países Andinos, la Organización Latinoamericana y del Caribe de Centros Históricos (OLACCHI), el Ministerio de Desarrollo Urbano y Vivienda (Miduvi) y ONU-Hábitat Ecuador, organizaron el Seminario Internacional «Jerarquías urbanas: ¿una pirámide trunca?», que se desarrolló en la sede del IAEN, en Quito, los días 3 y 4 de junio de 2013. El seminario formó parte de un programa apoyado por la Cooperación Regional para los Países Andinos y el IFEA sobre el tema «Las Ciudades Sostenibles y la Lucha contra la Pobreza», desarrollado en Colombia, Ecuador, Perú y Bolivia. Dentro de la programación del Seminario Internacional, se llevaron a cabo un conjunto de conferencias y mesas de debate por parte de académicos internacionales. Asistieron unas 300 personas durante los 2 días del evento. 
El seminario empezó con un homenaje a Saskia Sassen, premio Príncipe de Asturias de Ciencias Sociales 2013 y al lanzamiento de su libro «Ciudad y globalización» en la colección «Textos urbanos» de OLACCHI dirigida por Fernando Carrión. Saskia Sassen dio enseguida una conferencia magistral: «Urbanizar la tecnología, ampliando su desafío». Explorando las relaciones entre ciudades y tecnologías, pone en evidencia la tensión entre el hecho de que, por un lado, grandes compañías tecnológicas están vendiendo sistemas técnicos masivos para manejar aspectos claves de las ciudades y por otro lado, las ciudades siguen siendo sistemas incompletos, lo que abre la posibilidad de crear — crear lo urbano, lo político, lo cívico, una historia-. Muchas tecnologías no han sido suficientemente «urbanizadas», es decir que encierran los utilizadores en ciertos usos predefinidos que rompen con el abanico de posibles que ofrece de la vida urbana. Por lo tanto, Saskia Sassen aboga por construir nuevos usos tecnológicos que hagan posible un urbanismo de conocimiento abierto (open source) para aquellos aspectos técnicos de las ciudades donde esto pueda darse.

La temática de las jerarquías urbanas se refiere a un enfoque de los estudios urbanos muy desarrollado durante la época de crecimiento rápido (1950-1990), enfoque que enfatizaba en el caso latinoamericano, la concentración de la población en muy pocas ciudades (macrocefalia urbana) y de concentración de la población en las periferias de nuestras ciudades (cinturones de pobreza). En ese momento, la reflexión fue principalmente sobre el tema demográfico ya que la primacía urbana fue tan significativa que estableció una gran diferenciación entre la ciudad principal y las demás, una pirámide trunca. Hoy en día el subcontinente tiene todavía algunos de los índices de primacía urbana más elevados del mundo: Buenos Aires, Lima, Managua, Montevideo, Ciudad de Panamá, San José de Costa Rica, Santiago de Chile y Santo Domingo cuentan con más del $25 \%$ de la población nacional total.

Estos procesos históricos de urbanización del subcontinente han sido, según los casos, reforzados o matizados por los efectos del proceso más reciente de metropolización, entendida ésta como la concentración de los flujos de capitales, bienes e informaciones, de las riquezas y de las personas en algunas ciudades que dominan funcionalmente las redes urbanas: las metrópolis. Asimismo, las metrópolis no se definen por criterios demográficos sino por una combinación de variables que reflejan la capacidad de mando de dichas urbes en el marco de una economía globalizada. Se debilitaron las formas tradicionales de las relaciones de las ciudades con sus entornos regionales: las migraciones ciudadcampo, tan importantes durante la fase de rápido crecimiento, han disminuido fuertemente. Al mismo tiempo, se fortalecieron las relaciones de las ciudades con otras ciudades, más o menos próximas, en el marco de una economía cada vez más tercerizada. Estos procesos no se dan en todas partes, en todas las ciudades, con la misma intensidad ni con las mismas formas. Algunas ciudades, alejadas de los flujos globales con dificultades para insertarse en la nueva economía, pueden presentar un estancamiento económico y demográfico e incluso a veces un decrecimiento, mientras que otras, con ciertas «ventajas 


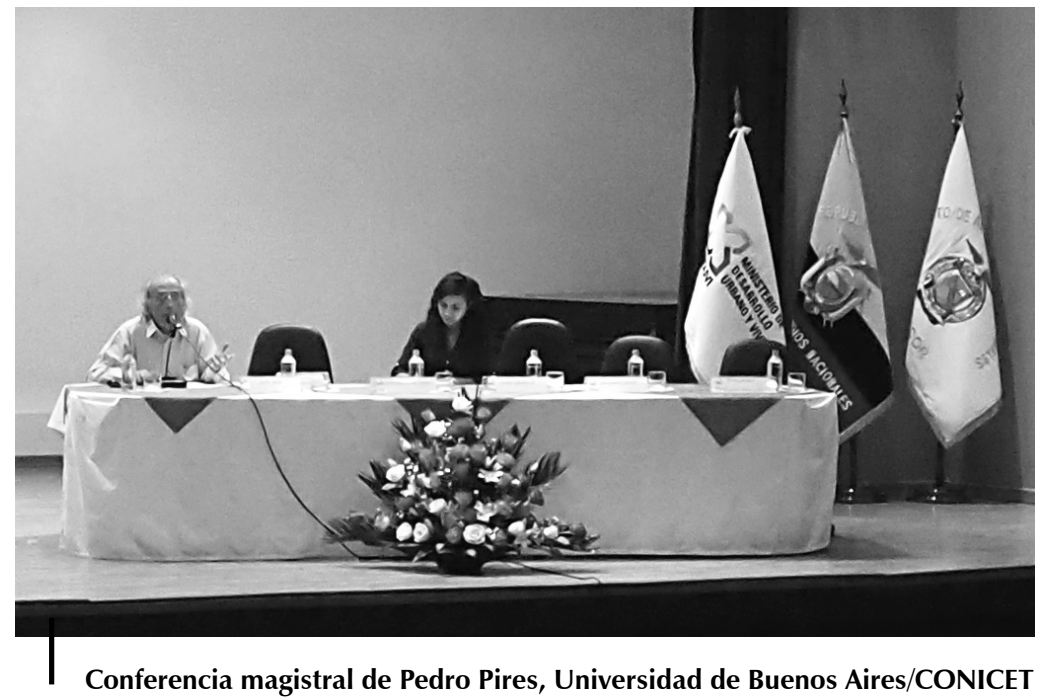

comparativas» específicas dentro del orden espacial de la globalización, conocen un fuerte dinamismo: ciudades turísticas, ciudades fronterizas o portuarias, ciudades con actividades productivas muy especializadas. El proceso de metropolización no significa siempre la «desaparición» de las ciudades intermedias, como bien lo demuestra el dinamismo de muchas ciudades de esta categoría en América Latina.

Por lo tanto, este seminario tuvo como propósito discutir si efectivamente estamos dejando atrás esta idea de la jerarquía sobre la base de una pirámide trunca y si estamos entrando a una nueva fase, más compleja, donde se redefinen los roles y funciones de las ciudades, grandes, intermediarias y pequeñas dentro de los sistemas de ciudades de escala regional o nacional. Además del reto científico de entender mejor los procesos en cursos, esta situación plantea unos retos muy importantes en términos de gestión territorial. Varios países de América Latina, como Ecuador o México, están redefiniendo la organización político-administrativa de sus territorios nacionales y por lo tanto, necesitan saberes expertos para identificar las competencias por descentralizar y el grado de autonomía por otorgar a las ciudades, según sus rangos. Pero ¿cómo definir estos rangos de ciudad en el contexto actual?

El seminario se organizó entonces sobre la base de tres grandes mesas: metrópolis, ciudades intermedias y ciudades pequeñas. Cada una fue dividida en una conferencia principal y una mesa redonda. Pedro Pírez (Universidad de Buenos Aires, Argentina), Fernando Carrión (Flacso, Ecuador) y Alice Beuf (IFEA, Colombia) presentaron ponencias que resaltaron las funciones, dinámicas y la especificad de gestión de las metrópolis que estudian. Más precisamente cuestionaron la formación de la metrópoli de Buenos Aires, el carácter mundial de la metrópoli de Quito y el rol del tamaño de la ciudad en cuanto a la gestión 


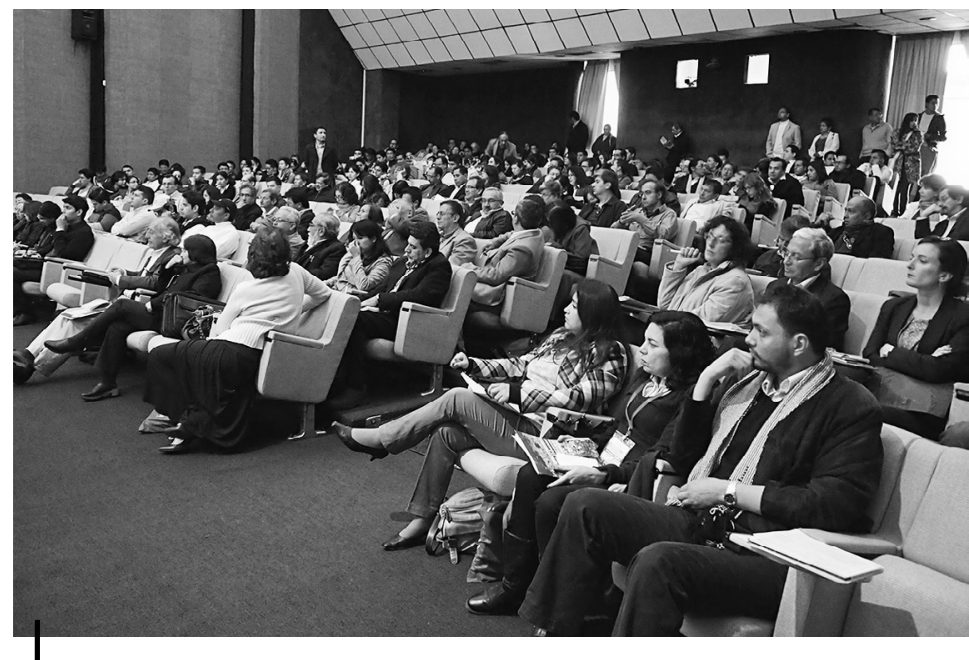

Seminario «Jerarquías urbanas», primer día por la tarde

urbana - amenaza u oportunidad - en el caso de Bogotá. La mesa sobre ciudades intermedias se llevó a cabo gracias a las contribuciones de Ricardo Jordán (Cepal, Chile), Graciela Fernández de Córdoba (Pontificia Universidad Católica de Perú) y Roxana Tapia Uriona (Cátedra Unesco «Ciudades intermedias: urbanización y desarrollo», Bolivia/España). La primera intervención de Ricardo Jordán brindó un balance de la gestión económica y urbana de este tipo de ciudades en América Latina, mientras que las dos siguientes presentaron estudios de caso que cuestionaron la manera cómo se define el carácter intermedio de las ciudades: un estudio sobre los valles costeños y andinos del Perú y una investigación sobre los valles de Cochabamba en Bolivia, en la cual se definen las ciudades intermedias como un recurso clave para el equilibrio territorial. La mesa sobre ciudades pequeñas la conformaron Jorge Mario Aponte (Universidad Nacional de Colombia) y Doralice Sátyro Maia (Universidade Federal de Paraiba, Brasil). El primer ponente presentó el caso de la construcción de un espacio urbano fronterizo, a partir de una mirada a Leticia y Tabatinga, dos ciudades pequeñas fronterizas en la Amazonía. La segunda se preguntó si los tiempos lentos, la relación con el campo y la escala son características en transición o en mutación de las pequeñas ciudades, a partir de cuatro ejemplos de pequeñas ciudades en distintos estados de Brasil.

El seminario dejó también un espacio para la reflexión sobre los procesos de reforma territorial que implican, desde el Estado, la redefinición del rol de cada rango de ciudad. Así, Juan Fernando García, representante del Consejo Nacional de Competencias de Ecuador, presentó los procesos de descentralización, autonomía y formación de Distritos Metropolitanos Autónomos de Ecuador. Asimismo, Alfonso Iracheta (El Colegio de México) analizó el proceso de reforma urbana en dicho país. 
La diversidad de las ponencias presentadas demostraron la complejidad del tema de la redefinición de las jerarquías urbanas y la pertinencia de esta multiplicidad de enfoques. Se espera publicar las memorias del seminario para recoger estos aportes y contribuir a la reconstrucción de este campo de investigación en América Latina.

Alice BEUF 